\title{
Application Of XML For Telemetry Data Management
}

\author{
Zhiguo Zhang \\ Section 94, PLA 91550 \\ Dalian, China \\ 602544@sohu.com
}

\author{
Lei Gong, Hongping WANG \\ Section 94, PLA 91550 \\ Dalian, China \\ gl_leo@163.com
}

\begin{abstract}
At present, the telemetry data is stored and released in the form of binary file or text file, and the user can't find out the data structure from the file itself, this bring difficulties in query and the information sharing. To deal with the problem, a XML-based telemetry data management method was proposed by taking the advantages of extensible makeup language (XML). Large scale of telemetry data can be stored in sections using this method, index efficiency of telemetry data is increased obviously in the operation of practical system. The distribution ability of telemetry data is increased too.
\end{abstract}

Keywords- telemetry; XML; data management; data storage

\section{INTRODUCTION}

The results of telemetry data process is an important basis for the performance evaluation in weapon testing. As the main product of weapon experiment, the telemetry data play a crucial role in weapon system development, identification and simulation analysis [1]. Due to the lack of efficient and advanced data management tools, there exist a lot problem in the maintenance and use of data, such as data integrity, data consistency, security and so on, these problem make the further tap of the data very difficult. So, the data management method seriously hampers the weapon test efficiency and innovation.

At present, the telemetry data is stored and released in the form of binary file or text file, and the user can't find out the data structure from the file itself, this bring difficulties in query and the information sharing by network of telemetry data [2]. With the rapid development of Internet technology and the increasingly strong demands for standardization of data exchange, telemetry data processed online and released through Web will greatly improve the efficiency of data processing and information sharing. Therefore, a suitable telemetry data format, which provides convenient data structure for online processing and Web release is needed. In this paper, we discuss the characteristics of XML (eXtensible Markup Language), and prove that XML is a suitable data format for data exchange and online data processing.

\section{THE CHARACTERISTICS OF XML}

XML, established by W3C, is designed as a mixed programming language in order to transfer information between user and software [3]. It has a series of excellent features, such as extendibility, simplicity, self-describing, and separation of structure, content and shell and so on. As it provides great flexibility for developers, XML has received strong support by free software programmer and commercial software industry.

Since XML data is stored in plain text format, XML provides a software and hardware-independent way of sharing data [4]. This makes it much easier to create data that different applications can work with. It also makes it easier to expand or upgrade a system to new operating systems, servers, applications, and new browsers. XML can also be used to store data in files or in databases. Applications can be written to store and retrieve information from the store, and generic applications can be used to display the data [5]. With $\mathrm{XML}$, your data is available to more users.

XML provides two useful tools for data modeling: DTD and XML Schema. In using these tools, developers can make standards for a group of document with same logical structure, rather than just one single document [6]. By this way, XML make documents more intelligent, such as providing better error checking function, make the extraction of useful information easier, and display the data accordance with the needs of user.

XML organize the data in the form tree view. A wellformed and valid XML document will establish a tree in memory after parsing by DOM. Therefore, the XML model maintains the order and structure of data after analysis by XML document parser, which formed in real-world data semantics and hierarchy. This character of XML makes the development easier to the developer. Meanwhile, the maintenance of the data formed in this structure is relatively simple.

In addition, the XML parser is a component with standard interface, thus avoiding duplication of development and distribution of the interface program, as well as reducing the corresponding interface program testing and maintenance cost.

This paper makes full use of the advantage of XML data management, solve the problems existing in the telemetry data management, and also improve the capacity of the telemetry data release. The method proposed in this paper proved to be a better telemetry data management method by experiment test.

\section{DATA MANAGEMENT BASED ON XML}

\section{A. Ideas}

XML elements can have attributes in the start tag. Attributes are used to provide additional information about elements. 
This paper uses XML document to organize the attribute and value of telemetry parameters. The attribute information includes the name of the parameter, ID, units, sample rate, start time, end time, etc. The data contains time codes and physical quantities. In order to achieve a valid query, appropriate XML search engine is needed. The search engine simplifies the data structure while meets the need for a query. Based on the data model presented above, the telemetry data management system designed as follows:

$<$ ?xml version='1.0' encoding='GB2312' standalone='yes'? $>$ $<$ Para Info $>$

$<$ Name $>$ Voltage $<$ / name $>$

$<$ Code $>$ Us $<$ /code $>$

$<$ Unit $>$ V $</$ Unit $>$

$<$ Freq $>40</$ Freq $>$

$<$ Start $>0</$ start $>$

$<$ End $>289.5</$ end $>$

$</$ Para Info $>$

$<$ Data $>$

$<$ Time $>0.025</$ time $>$

$<$ Value $>29.85<$ / value $>$

$</$ Data $>$

There is a variety classification method of telemetry parameter. Usually, the parameter is divided into fastvarying parameters and slow-varying parameters according to sample rate, electricity parameters and non-electricity parameters according to the sample-code method. To meet data management needs, this paper employ two means in telemetry data storage, point-by-point storage and feature segments storage. Generally, the amount of data stored by feature segment is relatively large, for example, a highfrequency vibration parameter may reach $300 \mathrm{M}$ when stored by feature segment, and $10 \mathrm{M}$ when stored point-by-point. In order to improve the efficiency of data retrieval, this paper adopts different management strategies to the point-by-point data and feature segments data respectively.

\section{B. Management of Point-by-Point Data}

In the procedure of data processing, data is extracted by point as the data amount of point-by-point parameters is relatively small. In this way, a single point-by-point parameter can be stored as a single XML document. The property information of the parameter is stored in this XML document as well as the data. Each field $<$ Data $>$ markup a sample-point data, and the property $<$ Time $>$ represents the sampling moment while the property $<$ Value $>$ represents the physical value of the parameter at the sampling moment.

To get the time and value in XML document, use the following function:

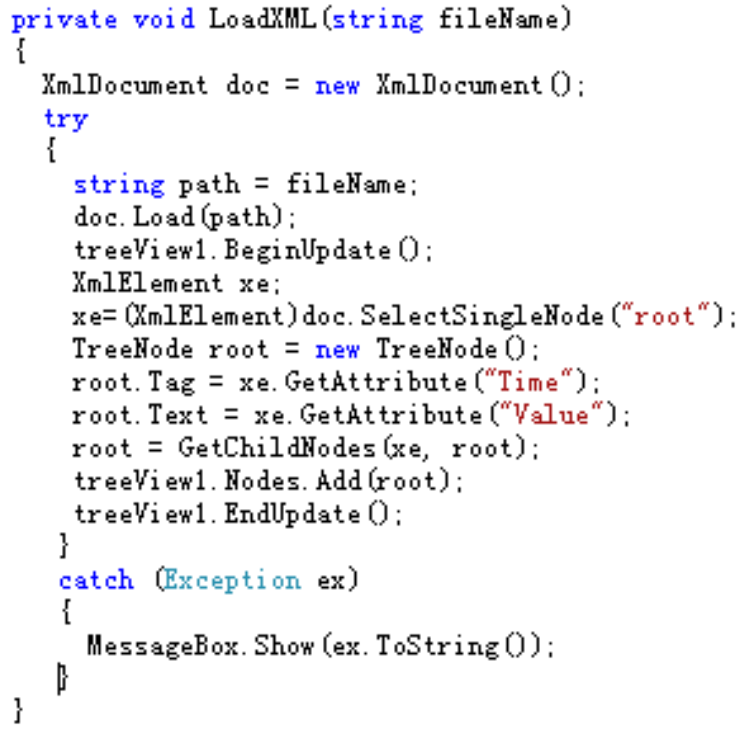

Figure 1. Code sample

\section{Management of Feature Segment Data}

The structure of feature segment data is the same as point-by-point data, but the amount of data is much greater. If stored like a point-by-point data, the XML document will be very large. Generally, we only care about the feature segment of the data, so, it's no need to store the data pointby-point. In addition, the search engine of XML can only search documents in order, so, when the feature segment lies close to the end of the data, the search efficiency will be very low. Therefore, a special storage format is required for feature segment parameters.

In this paper, we proposed a method named Sub-Feature Segment (SFS) for the storage of feature segment data. First, feature segment parameter was separated into a number of segments on the basis of characteristic moment, according to the application requirements. Then, each segment was stored in the form of XML document, just like point-bypoint data. Segment information was added into the field $<$ Data $>$ to distinguish each segment. Compare to the pointby-point file, three fields named $<$ Frag Name $>$, $<$ Start $>$ and $<$ End $>$ are put in the feature segment XML file. The field $<$ Frag Name $>$ represents the information of the feature segment data, the field $<$ Start $>$ indicates the start time of the data and the field <End $>$ indicates the termination time of the data. The data format of fast-varying parameter can described as follows:

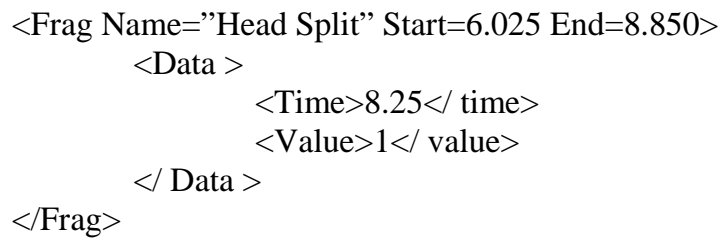


When the user needs to find a specific segment of the parameter, the field <Start>, <End $>$ and the search condition was get into account. XML search engine retrieve the data marked by XML, and finally find the needed segment.

Segment particle size influences the retrieval speed, segment particle size is too large, data retrieval efficiency will be reduced, segmented particles is too small, system needs to retrieve the data of multiple segments and stitching data, and it also will reduce the Retrieval efficiency. Therefore, it is necessary set the appropriate segment particle size according to the application requirements.

In use of this segmented storage strategy, search engine only needs to retrieve the segment data matching the search conditions, but not retrieve the whole time-course data in order, and greatly improve the retrieval speed of fastvarying parameters.

\section{EXPERIMENTS AND ANALYSIS}

In our experiment, we apply the method described above to the telemetry feature segment data. In order to show the efficiency improved by this method, we retrieve segmented data and un-segmented data separately. The experiment test the time required in searching $1 \mathrm{M}$ data under both conditions of segmented and un-segmented when the needed segment lies in different position of the data. The experiment environment was as follows:

TABLE I. EXPERIMENT ENVIRONMENT

\begin{tabular}{|c|c|c|c|}
\hline $\begin{array}{c}\text { programming } \\
\text { language }\end{array}$ & CPU & Memory & Data Size \\
\hline C\# & $\begin{array}{c}\text { Pentium } \\
2.4 \mathrm{G}\end{array}$ & $2048 \mathrm{M}$ & $100 \mathrm{M}$ \\
\hline
\end{tabular}

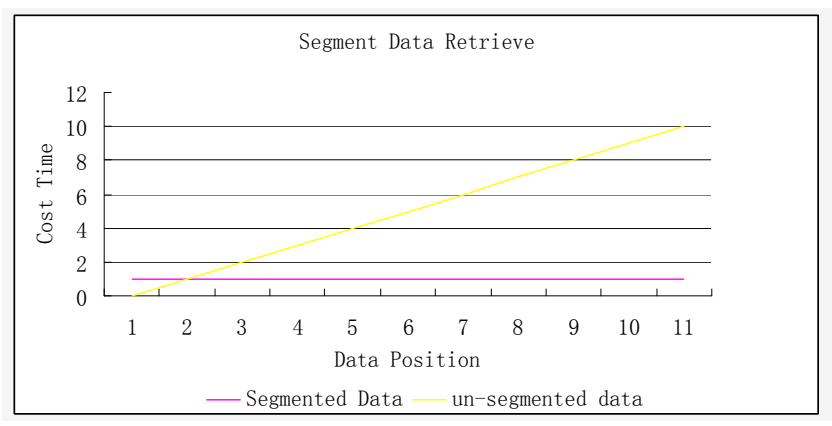

Figure 2. Feature segment data comparison

We compare the retrieve efficiency of the two data storage model, segmented data and un-segmented data, with the same query algorithm. Figure 1 shows the result of the experiment. The $\mathrm{X}$-axis of Figure 2 is position of the required data segment, and the $\mathrm{Y}$-axis is the time spends to find the required data. As can be seen from Figure 1, the time spend on data retrieving get longer with the location of the required data get closer to the end in the condition of unsegmented data, the search efficiency get worse; to the contrary, data retrieval time is almost independent to the location of the required data in the segment condition, and the retrieval efficiency is fixed.

But to the Point-by-Point Data, the retrieve efficiency of the two data storage model, segmented data and unsegmented data is almost the same. Figure 3 shows the result of the experiment.

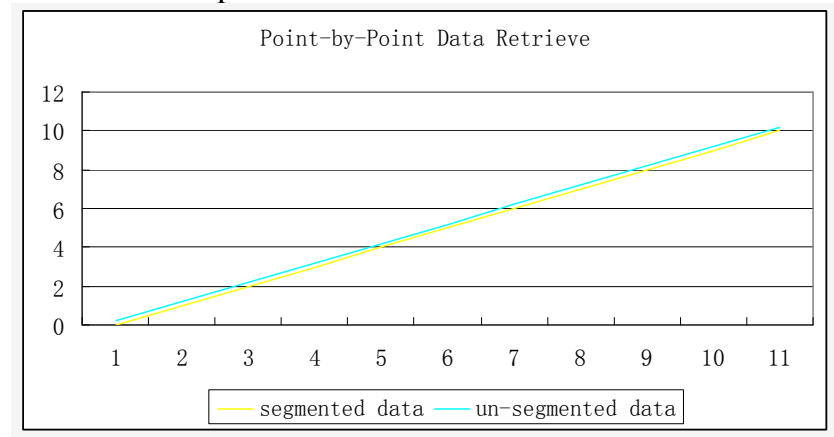

Figure 3. Point-by-Point data comparison

\section{CONCLUSION}

To solve the problem of telemetry data management, the XML-based method is proposed by combining the advantages of XML with database system. Based on XML storage, large scale of telemetry data can be stored in sections, index efficiency of telemetry data is increased obviously in the operation of practical system. Due to the inherent advantages of XML documents in the Internet data distribution, we can send the unparsed XML document to the client directly. Clients can take advantage of XML documents to do more works, such as analyzing, handling, etc. So, the distribution ability of telemetry data is increased too using this method.

\section{REFERENCES}

[1] Yin-Wen Qiu, "Research and Implementation of a General Missile Telemetry Data Processing Platform," Shang hai University, vol. 06, pp. 34, 2008.

[2] Zhang Chenguang and Lü Ming and Wang Gang, "Research and Implementation of a General Missile Telemetry Data Processing Platform ,” Missiles and Space Vehicles, vol. 02, pp. 45, 2005.

[3] Feng Jin and Ding Bo and Shi Dian-xi and Zhang and Zhu-xi and Xu Kai, "Research on the XML Parsing Technology ," Computer Engineering \& Science, vol. 02, pp. 23, 2009.

[4] Zhang De-hua, "Design and Implementation of Information Exchange System Based on XML,” Computer \& Network, vol. 01, pp. 15, 2011.

[5] Liu Ping-feng, "Method of intelligent recommendation of Web service resources towards users' balanced requirements," Application Research of Computers, vol. 09, pp. 42, 2012.

[6] Cao Lan ying and Yan Yi and Wu Huifeng, "Automating XML document transformations based on schema matching," Computer Engineering and Applications, vol. 25, pp. 37, 2012. 